\title{
Elastomer based electrically tunable, optical microcavities
}

Irma Slowik ${ }^{1,4}$, Nils M. Kronenberg ${ }^{2}$, Markus Franke ${ }^{3,4}$, Axel Fischer ${ }^{1}$, Andreas Richter ${ }^{3,4}$, Malte C. Gather ${ }^{2}$ and Karl Leo ${ }^{1,4}$

${ }^{1}$ Institut für Angewandte Photophysik, Technische Universität Dresden, George-Bähr-Strasse 1, D-01069

Dresden, Germany

${ }^{2}$ SUPA, School of Physics and Astronomy, University of St Andrews, North Haugh, St Andrews KY16 9SS, Scotland, UK

${ }^{3}$ Institut für Halbleiter- und Mikrosystemtechnik, Polymere Mikrosysteme, Technische Universität Dresden, D-01062 Dresden, Germany

${ }^{4}$ Center for Advancing Electronics (cfaed), Technische Universität Dresden, D-01062 Dresden, Germany

Tunable optical elements are mostly realized by microelectromechanical systems, which require expensive and complex lithography during processing. We demonstrate an alternative device based on an electrically tunable microcavity employing a dielectric soft elastomer actuator. The cavity resonance is varied by changing the physical cavity thickness due to electrostriction of the soft elastomer. We realize a tunable metal-elastomerDBR multi-half wavelength microcavity with a cavity layer thickness around $12 \mu \mathrm{m}$ and quality factors up to 700. Applying a voltage up to $60 \mathrm{~V}$ between bottom ITO and top metal electrode tunes the wavelength of the cavity modes up to $\Delta \lambda=14 \mathrm{~nm}$, which relates to a cavity thickness change of about $200 \mathrm{~nm}$. This concept allows the implementation of tunable optical elements like tunable filters or resonators with low cost and simple processing.

Electrically tunable optical microcavities are used for versatile applications in the field of sensing, spectroscopy as well as telecommunications ${ }^{1}$. Particularly for biomedical applications or chemical point-of-care analysis, it is desirable to develop simple, compact, and easily processable devices. For this purpose, optical sensing promises high sensitivity and a reduced response time ${ }^{2}$. Reconfigurable filters with small bandwidth were realized up to now by microelectromechanical systems (MEMS $)^{314}$ which require complicated etching and lithography or the application of thin membranes as tunable elements. By comparison, our approach is based on tuning microcavity resonance via an electroactive elastomer, is easy and cost-effective. Due to simple design, various structures and scaling to large area arrays are envisioned.

Dielectric elastomers (DE) are promising materials for electromechanical systems because of their ability to deform reversibly under applied voltage up to very high strains ${ }^{6}$. Recent applications include artificial muscles $\frac{6}{6}$, loudspeakers ${ }^{7}$, stretchable integrated circuits ${ }^{8}$, and generators $\$$. Due to their high transparency and flexibility, they show high potential to realize tunable optical elements like tunable phase plates ${ }^{10}$, gratings ${ }^{11}$, lenses,$\frac{12113}{12}$ or microcavities ${ }^{4}$.

Fig. 1] shows the operation principle of a dielectric elastomer actuator. When an external voltage $V$ is applied, the elastomer film gets squeezed due to the electrostatic pressure. Pelrine et al ${ }^{14}$ demonstrated that in a plate capacitor set-up, the effective pressure $p$ is written as:

$$
p=\varepsilon_{0} \varepsilon_{r} E^{2}=\frac{\varepsilon_{0} \varepsilon_{r} V^{2}}{z^{2}}
$$

where $\varepsilon_{0}$ is the dielectric constant, $\varepsilon_{r}$ the relative permittivity, $E$ the electric field strength and $z$ the thickness of the elastomer film. In the simplest model for an unloaded, unconstrained actuator, the thickness strain is

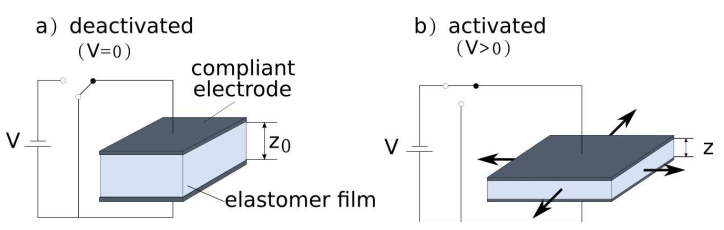

FIG. 1. Operation principle of a dielectric elastomer actuator: (a) A deactivated elastomer film with initial thickness $z_{0}$ is sandwiched between two compliant electrodes. (b) Under applied voltage the elastomer reduces thickness by $\Delta z$ and expands in lateral dimensions.

calculated by Eq. 1 and the elastic modulus $Y$, which is defined as ratio of stress $\sigma$ to strain $s$, so that $\sigma=Y s$. For small strains up to $10 \%$, it can be assumed that $z \approx z_{0}$, where $z_{0}$ is the initial thickness of the elastomer film 15 . Hence, the thickness variation $\Delta z$ is described for small strains by:

$$
\Delta z=\frac{\varepsilon_{0} \varepsilon_{r} V^{2}}{Y z_{0}}
$$

As the dielectric elastomer used in this work is incompressible, flexible electrodes are essential for the realization of the actuator. Compliant metal electrodes on soft elastomers are rather difficult to achieve due to the large difference in the elastic modulus of the used elastomer ( $\mathrm{kPa}$ range) and the metal (tens of $\mathrm{GPa}$ ). Furthermore, rigid substrates influence the actuator performances due to the one-side-constraint of the soft-dielectric film 21. Particularly on thin elastomer films, a metal electrode is expected to have a significant stiffening impact on the structure. Also, the low elasticity of metals $(2-3 \%)^{\sqrt{8}}$ hampers the usage of metal electrodes as cracks are formed during stretching of the elastomer film $[19$. Improvements in the elasticity of metal films were achieved via structuring of the electrodes 
either in zig-zags $\frac{15}{}$, coils $\frac{16}{16}$, or as horseshoe shaped tracks $\frac{17}{17}$. An alternative means to keep the stiffening impact of the metal electrode low but at the same time maintain a closed film with reasonable conductivity, is to reduce the lateral dimension of the metal layer $\frac{18}{\text {. }}$.

Here, we report on metal-elastomer-DBR microcavities as schematically illustrated in Fig. 2 (a). The (a)

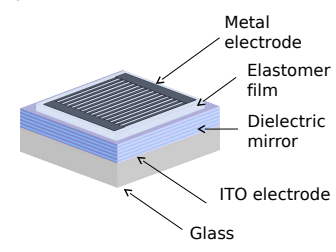

(b)

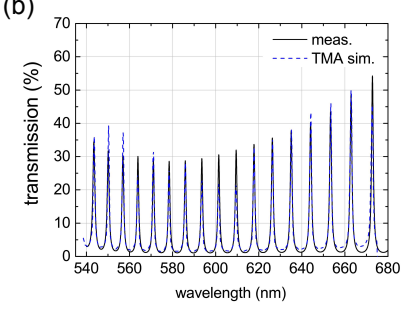

FIG. 2. (a) Schematic picture of the sample design of the electrically tunable metal-elastomer-DBR microcavity. A ITO film on the glass substrate acts as transparent bottom electrode. On top a highly reflecting DBR mirror is deposited with 21 alternating layers of $\mathrm{SiO}_{2}$ and $\mathrm{TiO}_{2}$. The soft elastomer layer acts as tunable cavity layer. Structured metal electrodes are thermally evaporated acting as top electrode and highly reflecting mirror. (b) Transmission spectra of a metal-elastomer-DBR microcavity with a cavity layer thickness of about $15.3 \mu \mathrm{m}$ measured within in the stop band of the DBR. Fabry-Perot modes are formed with quality factors up to 700 defined by the reflectivity of the DBR and the metal mirror. The blue dashed line depicts the corresponding transmission spectra generated by transfer matrix algorithm.

bottom mirror is formed by a distributed Bragg reflector (DBR) made of 21 alternating layers of $\mathrm{SiO}_{2}$ and $\mathrm{TiO}_{2}$ deposited on a glass substrate with $\approx 90 \mathrm{~nm}$ of indium tin oxide (ITO) layer serving as the bottom electrode. A film of an ultra-soft poly(siloxane) based elastomer (stiffness $\approx 3 \mathrm{kPa}$ ) produced by spin coating and heat curing is deposited on the DBR. By variation of the rotation speed, the thickness is adjusted between 3 and $15 \mu \mathrm{m}$. To increase the surface energy of the elastomer layer, a soft oxygen plasma treatment is performed prior to the physical vapor deposition. The top mirror and electrode is formed by a $25 \mathrm{~nm}$ thick silver layer, which is deposited on the elastomer layer by physical vapor deposition. The film thickness used here represents a trade off between good mechanical compliance on the one hand side and high optical reflectance and electrical conductivity. Depending on application, adjustments with better mechanical or optical performance would be possible. Fig. 2 (b) depicts exemplary a transmission spectra of an metal-elastomer-DBR microcavities with a layer thickness of $15 \mu \mathrm{m}$. The measured spectra is compared with a simulation by transfer matrix algorithm showing a good agreement between both. A Fabry-Perot cavity is formed between the dielectric bottom mirror and the top metal layer. The width of the peaks depends on quality factor of the cavity and thus on the reflectivity of both mirrors. The mode position and the distance is defined be the cavity layer thickness. Reducing the optical thickness of the cavity layer leads to a shift of the mode positions to lower wavelengths as well as in a decrease of the free spectral range.

Due to the low surface energy of untreated siloxane elastomers, thermally deposited silver tends to grow in nano-islands, form clusters and microcracks22]. Such non-uniform metal films with high roughness exhibit a lower reflectivity and an enhanced absorption by surface plasmons. To overcome this problem, the elastomer is treated with a soft oxygen plasma prior to the metal deposition which increases the surface energy by increasing the number of hydrophilic groups on the polymer surface $^{23}$. By applying oxygen plasma treatment, we are able to double the reflectivity of the metal film and thus achieve quality factors for the multi-half wavelength microcavity up to 700 (compare Fig. 2 (b)).

Electrostriction of the metal-elastomer-DBR cavity is tested by optical investigations. Fig. 3 (a) shows confocal microscope image of the edge of the metal electrode under monochromatic illumination at $650 \mathrm{~nm}$ at various applied voltages. Differences in optical thickness of the device are visible as interference fringes. Applying a voltage reduces the microcavity thickness and therefore interference structure. The minimum in the interference pattern at the edge of the electrode changes to a maximum, which refers to a variation of optical thickness of $\lambda_{0} \backslash 4 n \approx 115 \mathrm{~nm}$. Due to the constraint of the elastomer on the rigid substrate, the active deformation occurs mainly at the rim of the top metal electrode where the compressed material expands to the metal free area. The variation of the cavity layer thickness leads to a change of the mode spectrum of the microcavity (Fig. 3 (b)). A change in the cavity spectrum under applied voltage is observed by reflection measurements close to the metal edge (Fig. 3 (c)). The quadratic dependence between applied voltage and cavity deformation (compare Eq. 2 is confirmed by fitting a simple quadratic function (Fig. 3 (d)).

To investigate the spatial distribution of the actuation, cross sections at the metal edge are analysed. Fig. 4 (a) depicts the profile of the actuated sample discussed in Fig. 3. The compression of the cavity underneath the electrode and the expansion outside the electrode area are phenomenologically described with a bi-Gaussian function for each peak (red dashed line). From this, the width of the deformation is estimated to be approximately $50 \mu \mathrm{m}$.

The performance of the actuator is further improved by structuring the top electrode, as depicted in Fig. 4 (b). In this case, narrow metal stripes (width, $90 \mu \mathrm{m}$ ) were realized by physical vapour deposition through a suitable shadow mask. The profile of the actuated device exhibits two maxima of deflection with around $35 \mu \mathrm{m}$ width while material is squeezed out of the electrode area. Therefore the cross section shows a 'W'-like shape. 
(a)

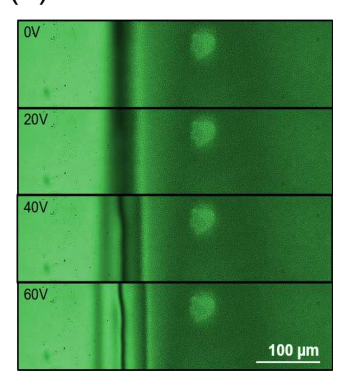

(b)

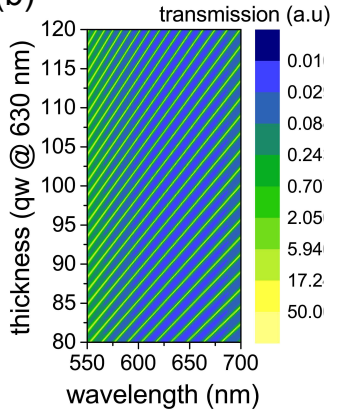

(c)

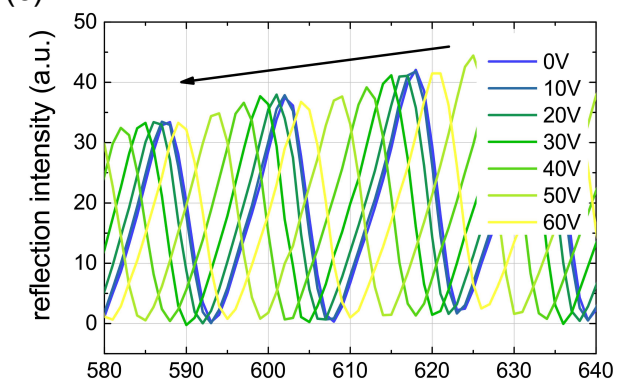

(d)

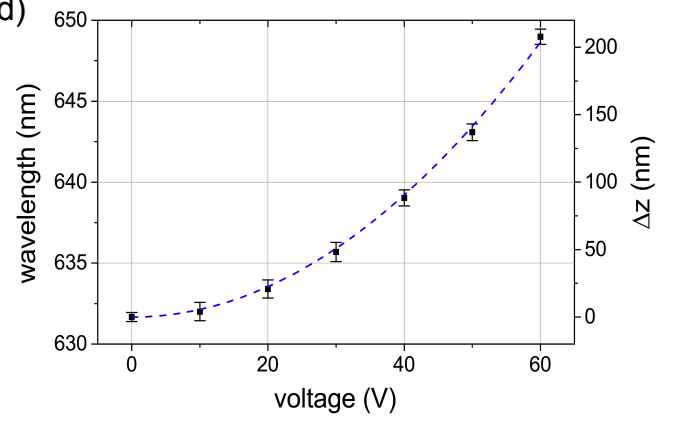

FIG. 3. Optical characterization of the metal-elastomer-DBR cavity ( $25 \mathrm{~nm} \mathrm{Ag}, 12 \mu \mathrm{m}$ elastomer): (a) Confocal microscope image of metal electrode edge under monochromatic illumination at $650 \mathrm{~nm}$ for various applied voltages. A change of interference fringes at the rim of the metal film indicates a thickness change at these positions. (b) Simulated transmission spectra of the metal-elastomer-DBR cavity with transfer matrix algorithm shows the shift of mode spectrum depending on the optical thickness of the cavity layer measured in quarter wavelength (qw) for a design wavelength of $630 \mathrm{~nm}$. (c) Reflection spectra measured for different applied voltages. (d) Change in position of a mode located around $633 \mathrm{~nm}$ and the decrease in cavity thickness derived from this shift as a function of the applied voltage (black dots). The blue dashed line represents a quadratic fit of the measured data using Eq. 2

However, the quality factors measured close to the metal edge are much lower than in the reference shown in Fig. 2 (b). This effect occurs because of the shadow mask evaporation not sharp but more trapezoid-like metal edges are formed. Higher quality factors with expense of the tuning range can be achieved with increasing dis-
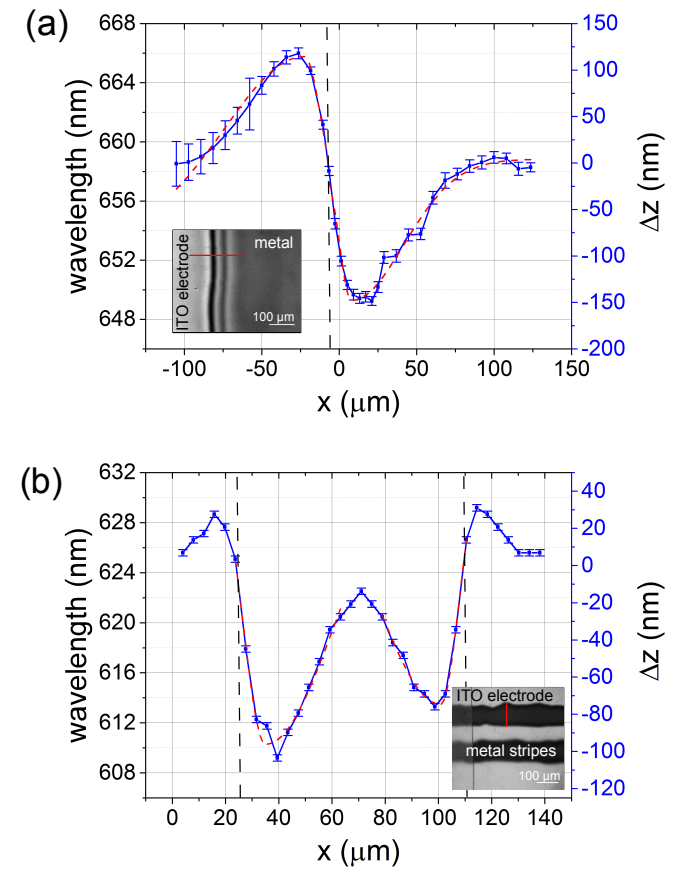

FIG. 4. (a) Cross section profile of the sample discussed in Fig. 3 shows actuation of metal electrode and the respective mode shift for an applied voltage of $60 \mathrm{~V}$. The edge of the electrode is marked in the profile a with black dashed line. The deformation is fitted with a bi-Gaussian function for each peak (red dashed line). Inset: confocal microscope image of sample. The cross section is taken at the red line. (b) For comparison, the profile for a sample with a structured electrode (stripe width, $90 \mu \mathrm{m}$ ) at the same voltage. The actuation has to maxima at the edges of the metal stripe resulting in a 'W'-like shape of the deformation.

tance to the edge. Hence, transmission spectra of the tunable cavity are measured taken with a distance of 50-100 $\mathrm{\mu m}$ to the metal edge for different applied voltages using a microscope setup (objectives: focusing $25 \mathrm{x}$ NA 0.5, imaging 50x NA 0.8) shown in Fig. 5 . Due to the non-homogeneous deformation of the elastomer layer (see Fig. 4 (a)) a smaller wavelength shift up to $1 \mathrm{~nm}$ is detected compared to the reflection spectra shown in Fig. 3 (c). Even so, because of the large spot size $(\approx 50 \mu \mathrm{m})$ a broadening of the modes occur for increasing external voltage. Therefore, the quality factor of the cavity drops from 700 to 300 at $90 \mathrm{~V}$ respectively. Nevertheless, the effect is less pronounced for smaller spot size or lower external voltages. Furthermore, the quality factor can be enhanced using thicker metal layers e.g. increasing the the thickness of the silver layer to $50 \mathrm{~nm}$ would roughly double the quality factor of the optical cavity. However, a thicker metal electrode leads to an higher stiffening impact on the soft elastomer and therefore will cause a lower tuning range of the cavity. On the other hand, the tunability can be increased using thin- 


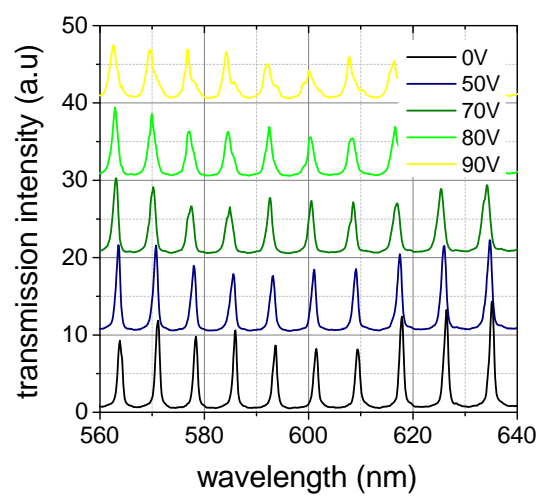

FIG. 5. Transmission spectra for the metal-elastomer-DBR cavity shown in Fig. 4 (a) measured in a microscope setup with a spot size of about $50 \mu \mathrm{m}$ (objectives: focusing $25 \mathrm{x}$ NA 0.5 , imaging $50 \mathrm{x}$ NA 0.8 ). The spectra are taken wit a distance of 50-100 $\mathrm{\mu m}$ to the edge. Under applied voltage the position of the optical modes is shifted up to $1 \mathrm{~nm}$ to lower wavelength because of the compression of the soft elastomer cavity layer. Due to inhomogeneous deformation of the elastomer layer, the quality factor decreases from $700(0 \mathrm{~V})$ to $300(90 \mathrm{~V})$.

ner cavity layers due to the higher electrical field strength (compare Eq. 2) at a small expense of the quality factor. However, for very thin elastomer layer the activated surface layer has an higher impact on the elasticity of the device. Even so, the stiffening impact of the metal layer increases for thinner layers. Therefore, the layer thicknesses of the different components has to be well chosen depending on the application of the optical cavity regarding their optical and mechanical properties.

Alternatively, electrical actuation is proven by capacitance measurements (see Fig. 6 (a)). Due to the decrease of the cavity layer thickness, the capacitance of the device increases. Assuming a plate capacitor set-up (compare Fig. 6 (b)), the capacitance $C$ can be written as:

$$
C_{0}=\frac{\epsilon_{0} \epsilon_{r} A}{z_{0}}
$$

Capacitance is measured by impedance spectroscopy assuming a simple RC element shown in Fig 6 (c). Since the DBR partly consist of the high-k material of $\mathrm{TiO}_{2}$, the capacitance $C_{\mathrm{DBR}} \approx 500 \mathrm{pF}$ can be neglected compared to the much lower value of the elastomer layer $C \approx 10 \mathrm{pF}$. Inserting Eq. 3 for the cavity thickness deformation results in a quadratic relationship between the applied voltages and the inverse capacitance. For this description, the inhomogeneous deformation of the elastomer layer has to be taken into account. By fitting the profile in Fig. 4 (b) with a bi-Gaussian function, the actual capacitance variation for $60 \mathrm{~V}$ is estimated to 1 - $3 \mathrm{fF}$ matching with the result of the electrical measurement of $2.2 \mathrm{fF}$. The capacitance measurements verifies the stability of the electrically tunable microcavity by

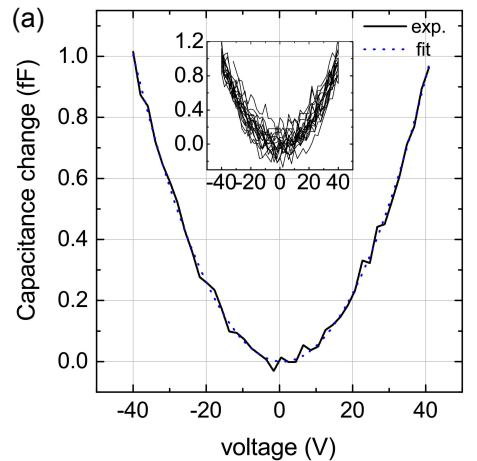

(b)

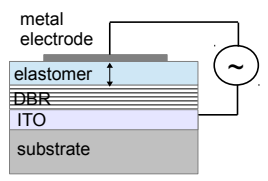

(c)

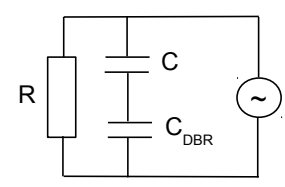

FIG. 6. (a) Averaged capacitance change $\Delta C$ under applied voltage $V$ measured by impedance spectroscopy $(f=$ $100 \mathrm{kHz}, V_{r m s}=200 \mathrm{mV}$ ) for 10 cycles. Following Eq. ?? the inverse capacitance follows a quadratic dependence on the applied voltage (blue dashed line). The inset shows the capacitance for 5 sweeps from -40 to $40 \mathrm{~V}$. (b) Schematic view of the measured sample structure; (c) Sample is described as a parallel RC element, while the capacitance of the DBR can be neglected.

more than 100 cycles of reversible actuation.

In conclusion, we have demonstrated an electrically tunable, optical filter realized by a tunable metalelastomer-DBR multi-half wavelength microcavity employing a thin film dielectric soft elastomer actuator. Applying a voltage between a bottom transparent electrode on the glass substrate and the top metal layer results in a deformation of the soft elastomer. We observed a reversible mode shift of the cavity modes up to $14 \mathrm{~nm}$ related to a cavity thickness change of about $200 \mathrm{~nm}$. The electrostriction follows a quadratic voltage behaviour, allowing a control of the resonant wavelength in a range below $1 \mathrm{~nm}$. Larger actuation can be achieved by using elastomers with lower Young's modulus as cavity layer or altering the metal layer thickness. Furthermore, the performance can be improved using structured electrodes reducing the stiffening impact of the metal eletrode on the soft elastomer layer. Since the devices are fabricated with low cost processing like physical vapor deposition and spin coating, the results offer the possibility to realize cost efficient and simple processable tunable optical elements, e.g. filters, resonators, or display application.

Acknowledgement: This work is supported in part by the German Research Foundation (DFG) within the Cluster of Excellence Center for Advancing Electronics Dresden (cfaed) and by the European Social Fund via the OrganoMechanics project. M.C.G. and N.M.K acknowledge support by the Scottish Funding Council (via SUPA) and the Human Frontier Science Program (RG0074/2013). 
${ }^{1}$ S. Forget and S. Chenais, "Organic Solid-State Lasers", Springer Series in Optical Science, Vol. 175, page 165 (2013)

${ }^{2}$ C. Vannahme, S. Klinkhammer, A. Kolew, P. J. Jakobs, M. Guttmann, S. Dehm, U. Lemmer, and T. Mappes, "Integration of organic semiconductor lasers and single-mode passive waveguides into a PMMA substrate", Microelectronic Engineering, 87, 693-695 (2010)

${ }^{3}$ M. Pruessner,T. Stievater, and W. Rabinovich, "Reconfigurable filters using mems resonators and integrated optical microcavities", Proc. of IEEE 21st International Conference on Micro Electro Mechanical Systems, 766-769 (2008)

${ }^{4}$ W. Chang, A. Wang, A. Murarka, G. M. Akselrod, C. Packard, J. H. Lang, and V. Bulović, "Electrically tunable organic verticalcavity surface-emitting laser", Applied Physics Letters, 105, 073303 (2014)

${ }^{5}$ W. C. Roentgen, "About the Changes in Shape and Volume of Dielectrics Caused by Electricity", ser. Annual Physics and Chemistry Series 11, Sec III, G. Wiedemann, Eds., J. A. Barth Leipzig, Germany 1880, p. 771.

${ }^{6} \mathrm{P}$. Brochu, and Q. Pei, "Advances in Dielectric Elastomers for Actuators and Artificial Muscles", Macromolecular Rapid Communications, 31, 10-36 (2010)

${ }^{7}$ R. Heydt, R. Kornbluh, J. Eckerle and R. Pelrine, "Sound radiation properties of dielectric elastomer electroactive polymer loudspeakers", Proc. SPIE, 6168, 61681M (2006)

${ }^{8}$ S. Rosset, and H. R. Shea, "Flexible and stretchable electrodes for dielectric elastomer actuators". Applied Physics A, 110, 281307 (2012)

${ }^{9}$ R. Pelrine, R. D. Kornbluh, J. Eckerle, P. Jeuck, S. Oh, Q. Pei and S. Stanford, "Dielectric elastomers: generator mode fundamentals and applications, Proc. SPIE, 4329 (2001)

${ }^{10} \mathrm{M}$. Beck, R. Fiolka, and A. Stemmer, "Variable phase retarder made of a dielectric elastomer actuator", Optics letters, 34, 8035 (2009)

${ }^{11}$ M. Aschwanden, D. Niederer, and A. Stemmer, "Tunable Transmission Gratings based on Dielectric Elastomer Actuators", Proc. of SPIE, 6927, 69271R-1 (2008)

${ }^{12}$ P. Liebetraut, S. Petsch, W. M"onch, and H. Zappe, "Tunable solid-body elastomer lenses with electromagnetic actuation", Applied optics, 50, 3268-74 (2011)

${ }^{13}$ C.-C. Huang, D. Liang, W.-P. Shih, and Z.-F. Lin, "Tunable lens driven by dielectric elastomer actuator with ionic electrodes", Micro \& Nano Letters, 9, 869-873 (2014)

${ }^{14}$ R.-E. Pelrine, R. D. Kornbluh, and J. P. Joseph, "Electrostriction of polymer dielectrics with compliant electrodes as a means of actuation", Sensors and Actuators A: Physical, 64, 77-85 (1998)

${ }^{15}$ R. Pelrine, R. Kornbluh, J. Joseph, R. Heydt, Q. Pei, and S. Chiba, "High-field deformation of elastomeric dielectrics for actuators", Materials Science and Engineering: C, 11, 89-100 (2000)

${ }^{16}$ A. Pimpin, Y. Suzuki, N. Kasagi, "Microelectrostrictive Actuator With Large Out-of-Plane Deformation for Flow-Control", Application Journal of Microelectromechanical Systems, 16, 753-764 (2007)

${ }^{17}$ M. Gonzalez; F.Axisa, M. V. Bulcke, D. Brosteaux, B. Vandevelde, Bart and J. Vanfleteren "Design of metal interconnects for stretchable electronic circuits", Microelectronics Reliability, 46(6), 825-832 (2008)

${ }^{18}$ F. M. Weiss, T. Töpper, B. Osmani, C. Winterhalter, and C. Müller, "Impact of electrode preparation on the bending of asymmetric planar electro-active polymer microstructures", Smart Structures and Materials, 9056, 905607 (2014)

${ }^{19}$ F. Habrard, J. Patscheider, and G. Kovacs, "Super-compliant metallic electrodes for Electroactive Polymer Actuators", Proc. of SPIE, 8340, 834013 (2012)

${ }^{20}$ D. Bodas, and C. Khan-Malek, "Hydrophilization and hydrophobic recovery of PDMS by oxygen plasma and chemical treatment - An SEM investigation", Sensors and Actuators B: Chemical, 123, 368-373 (2007)

${ }^{21} \mathrm{X}$. Zhao, and Q. Wang, "Harnessing large deformation and insta- bilities of soft dielectrics: Theory, experiment, and application", Applied Physics Reviews, 1, 021304 (2014)

${ }^{22}$ A. Thran, T. Strunskus, V. Zaporojtchenko and F. Faupel, "Evidence of noble metal diffusion in polymers at room temperature and its retardation by a chromium barrier, Applied Physics Letters, 81, 244 (2002)

${ }^{23}$ M. J. Owen, and P. J. Smith, "Plasma treatment of polydimethylsiloxane", Journal of Adhesion Science and Technology, 8, 1063-1075 (1994) 